\title{
Impact of dry eye on work productivity
}

This article was published in the following Dove Press journal:

ClinicoEconomics and Outcomes Research

9 October 2012

Number of times this article has been viewed

\section{Masakazu Yamada \\ Yoshinobu Mizuno Chika Shigeyasu}

National Institute of Sensory Organs, National Hospital Organization Tokyo Medical Center, Tokyo, Japan
Correspondence: Masakazu Yamada National Institute of Sensory Organs, National Hospital Organization Tokyo Medical Center, 2-5-I Higashigaoka, Meguro-ku, I52-8902, Tokyo, Japan

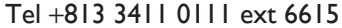

Fax $+8|334| I 0185$

Email yamadamasakazu@kankakuki.go.jp
Background: The purpose of this study was to evaluate the impact of dry eye on work productivity of office workers, especially in terms of presenteeism.

Methods: A total of 396 individuals aged $\geq 20$ years ( 258 men and 138 women, mean age $43.4 \pm 13.0$ years) were recruited through an online survey. Data from 355 responders who did not have missing values were included in the analysis. They were classified into the following four groups according to the diagnostic status and subjective symptoms of dry eye: a definite dry eye group; a marginal dry eye group; a self-reported dry eye group; and a control group. The impact of dry eye on work productivity was evaluated using the Japanese version of the Work Limitations Questionnaire. The cost of work productivity loss associated with dry eye and the economic benefits of providing treatment for dry eye were also assessed.

Results: The degree of work performance loss was 5.65\% in the definite dry eye group, $4.37 \%$ in the marginal dry eye group, $6.06 \%$ in the self-reported dry eye group, and $4.27 \%$ in the control group. Productivity in the self-reported dry eye group was significantly lower than that in the control group $(P<0.05)$. The annual cost of work productivity loss associated with dry eye was estimated to be USD 741 per person.

Conclusion: Dry eye impairs work performance among office workers, which may lead to a substantial loss to industry. Management of symptoms of dry eye by providing treatment may contribute to improvement in work productivity.

Keywords: burden of disease, dry eye, presenteeism, quality of life

\section{Introduction}

Although the prevalence of dry eye varies among the reports, it is widely recognized that dry eye is a chronic eye disorder that is highly prevalent in many countries, including Japan. ${ }^{1,2}$ Although the probability of dry eye causing blindness or permanent visual impairment is low, it is considered to have a significant impact on the daily and social lives of affected patients. ${ }^{3-5}$ Miljanovic et al reported that patients with dry eye syndrome had more difficulty reading, carrying out professional work, using a computer, watching television, and driving compared with those without dry eye. ${ }^{6}$ Utility assessment is a formal method for quantifying the relative impact of a given health state or disease on patient lives, which is defined on a continuous scale from 0 to 1 , where 0 corresponds to the worst possible quality of life weight (equal to death) and 1 corresponds to the best possible quality of life weight (equal to perfect health). Schiffman et al reported that the utility of moderate dry eye was 0.81 and that of severe dry eye was $0.72 .^{7}$ The burden of dry eye disease from both the prevalence and patient morbidity standpoints appears to be considerable. 
The disease burden can be divided into three categories, ie, direct costs such as medical fees; indirect costs such as low employment, absence from work, and impaired productivity; and a decrease in quality of life. Given that dry eye is highly prevalent among people of working age as well as in the elderly, its impact on work productivity is considered to be substantial. Moreover, it has been reported that the incidence of dry eye is particularly high in workers using visual display terminals, including laptops, tablets, electronic readers, and smartphones. ${ }^{8-10}$ Based on this background, it would appear worthwhile to evaluate work productivity in patients with dry eye, but few studies have been conducted from this viewpoint.

Loss of work productivity may occur through either absenteeism (absence, early leaving) or presenteeism. Presenteeism is a concept proposed by Auren in $1955^{11}$ to describe productivity loss when employees come to work but are not fully productive. In a study on the burden of dry eye disease, Reddy et al reported that patients with dry eye take 2-5 days off work annually, while they were present at work having symptoms for 191-208 days annually, indicating that presenteeism is a greater issue than absenteeism among those having dry eye. ${ }^{12}$

Patel et al conducted a study to evaluate presenteeism in patients with a diagnosis of dry eye using the Work Productivity and Activity Impairment Questionnaire, and found that those with higher scores of the Ocular Surface Disease Index, which were obtained by a questionnaire concerning subjective symptoms of dry eye, had a greater loss of work productivity. ${ }^{13}$ However, because this study did not include any subjects without dry eye, comparison of presenteeism against healthy individuals was not possible. In the present study, we evaluated the impact of dry eye on presenteeism using the Japanese version of the Work Limitations Questionnaire (WLQ-J, Sompo Japan Healthcare Services Inc, Tokyo, Japan) which is an established tool for evaluation of presenteeism. ${ }^{14-16}$ Furthermore, the cost of work productivity loss associated with dry eye and economic benefits of providing treatment for dry eye were also assessed.

\section{Materials and methods Subjects}

Using the general consumer panel (approximately 1,396,000 persons registered) run by Cross Marketing Inc (Tokyo, Japan), an Internet online survey was carried out targeting office workers aged $\geq 20$ years. The survey is based on the WLQ-J, a questionnaire consisting of 25 questions. Questions concerning the background of participants, such as age and gender, diagnostic status of dry eye, and 12 questions concerning their subjective symptoms were also asked. Of 618 individuals who initially showed an intention to participate in the study, 396 responded and answered the questions during the study period. The study period was between June 10, 2011 and July 4, 2011. Those who were engaged in medical services, eye-related industry, and market research were excluded from participating in the present study. The study was conducted in accordance with the Declaration of Helsinki of the World Medical Association, and the Ethical Guidelines for Epidemiology Research issued by the Ministry of Education, Culture, Sports, Science, and Technology and the Ministry of Health, Labour, and Welfare of Japan (July 17, 2002). The study was reviewed by a nonprofit organization, the MINS Institutional Review Board, through Nielsen Company Japan (Tokyo, Japan), which organized the questionnaire survey, and was conducted appropriately in accordance with their advice on the clinical study.

Participants were divided into subgroups according to their diagnostic status and subjective symptoms of dry eye. Those who had consulted an ophthalmologist in the past and received a diagnosis of dry eye were defined as having dry eye. Moreover, participants were asked whether or not they had subjective symptoms of dry eye within the past month according to the following 12 criteria: eyes get tired easily; have eye mucus; eyes feel gritty; eyes feel heavy; eyes feel dry; eyes feel uncomfortable; eyes feel painful; have tears without reason; have blurry eyesight; eyes feel itchy; feel the light dazzling; eyes are often reddened. Those who met more than five criteria were defined as having subjective symptoms according to the report of Toda et al. ${ }^{17}$ Based on the results, participants were classified into the following four groups: definite dry eye group, ie, those who have both symptoms and a diagnosis; marginal dry eye group, ie, those who have a diagnosis but no symptoms; self-reported dry eye group, ie, those who have symptoms but no diagnosis; and a control group, ie, those who have neither symptoms nor a diagnosis.

\section{Evaluation of work performance loss using WLQ-J}

Loss of work performance was evaluated using the WLQ-J, which is a questionnaire consisting of 25 items to estimate the degree to which health problems interfere with specific aspects of work performance and the impact of these work limitations on productivity by calculating scores using a specific algorithm. In addition to a total score to evaluate overall work performance, we also calculated subscale 
scores to evaluate four aspects of work limitations, ie, time management, and physical, mental/interpersonal, and output demands.

\section{Calculation of cost of work productivity loss and economic benefits of treatment}

Data on the average annual wage in Japan from the Basic Survey on Wage Structure 2010 released by the Japanese Ministry of Health, Labour, and Welfare ${ }^{18}$ and the degree of work performance loss associated with dry eye as measured by the WLQ-J were used to calculate the cost of work productivity loss due to dry eye. We previously estimated the annual direct cost incurred by dry eye patients, which includes the expenses for medical consultation, drug costs including over-the counter drugs, and cost of punctal plugs. ${ }^{19}$ The direct cost of dry eye in Japan was weighed against the cost of work productivity loss to evaluate the economic benefits of providing treatment for dry eye. Values in Japanese yen were converted to US dollars using the currency exchange rate as of June 26, 2011 ( 1 yen $=0.0124$ dollars).

\section{Statistical analysis}

Dunnett's test was used for comparison of the degree of work performance loss in each group, with a $P$ value of $<0.05$ considered to be statistically significant.

\section{Results \\ Participants}

A total of 396 individuals (258 men [65.2\%] and 138 women [34.8\%]) aged $43.4 \pm 13.0$ (mean \pm standard deviation) years responded to the survey. The age distribution was as follows: 175 (44.2\%) were aged 20-39 years; 170 (42.9\%) were aged $40-59$ years; and $51(12.9 \%)$ were aged $\geq 60$ years. Of the responders, 355 who did not have missing values were enrolled and classified into four groups according to a diagnosis and subjective symptoms of dry eye. Consequently, 69 were in the dry eye group, 128 were in the marginal dry eye group, 80 were in the self-reported dry eye group, and 78 were in the control group. There was no significant difference in terms of age or gender among the groups (Table 1).

\section{Impact of dry eye on work performance}

The degree of work performance loss according to diagnostic status and symptoms of dry eye was $5.65 \%$ in the definite dry eye group, $4.37 \%$ in the marginal dry eye group, $6.06 \%$ in the self-reported dry eye, and $4.27 \%$ in the control group. Work performance in the self-reported dry eye group was significantly lower than that in the control group $(P<0.05$, Figure 1). The subscale scores for the four aspects (time management, physical demands, mental/interpersonal, and output demands) were also calculated. Although the mental/ interpersonal score was significantly lower in the definite dry eye group $(P<0.05)$ and the self-reported dry eye group $(P<0.01)$, no significant difference was observed among the other groups (Figure 2).

\section{Cost of work productivity loss associated with dry eye}

The cost of work productivity loss associated with dry eye was calculated. The differences in work productivity compared with the control group was $1.38 \%, 0.10 \%$, and $1.79 \%$ in the definite dry eye group, the marginal dry eye group, and the self-reported dry eye group, respectively. Given that the average annual wage in Japan is currently USD 57,873 according to the Basic Survey of Wage Structure 2010 released by the Japanese Ministry of Health, Labour, and Welfare, ${ }^{18}$ the cost of work productivity loss per person in each group was calculated as USD 799, USD 58, and USD 1036 , respectively. Based on the difference in costs between the definite dry eye group and the control group, the cost of work productivity loss per person associated with dry eye was considered to be USD 799 .

Table I Background of participants $(n=355)$

\begin{tabular}{|c|c|c|c|c|c|}
\hline & $\begin{array}{l}\text { Definite dry } \\
\text { eye group }\end{array}$ & $\begin{array}{l}\text { Marginal dry } \\
\text { eye group }\end{array}$ & $\begin{array}{l}\text { Self-reported dry } \\
\text { eye group }\end{array}$ & Control group & $P$-value \\
\hline Participants (n) & 69 & 128 & 80 & 78 & \\
\hline Gender (men/women) & $4 I / 28$ & $87 / 4 \mid$ & $47 / 33$ & $57 / 21$ & $0.168^{b}$ \\
\hline Age (years) ${ }^{\mathrm{a}}$ & $42.9 \pm 12.4$ & $42.5 \pm 12.9$ & $42.8 \pm 12.5$ & $41.8 \pm 11.0$ & $0.944^{c}$ \\
\hline \multicolumn{6}{|l|}{ Age distribution (years) } \\
\hline $20-39$ & 31 & 63 & 37 & 33 & $0.289^{b}$ \\
\hline $40-59$ & 30 & 51 & 32 & 42 & \\
\hline$\geq 60$ & 8 & 14 & 11 & 3 & \\
\hline
\end{tabular}

Notes: ${ }^{2}$ Values are expressed as the mean \pm standard deviation; The following statistical analyses were used for comparisons between groups: ${ }^{\mathrm{b} C h i-s q u a r e ~ t e s t ; ~}{ }^{\mathrm{c} a n a l y s i s ~ o f ~}$ variance. 


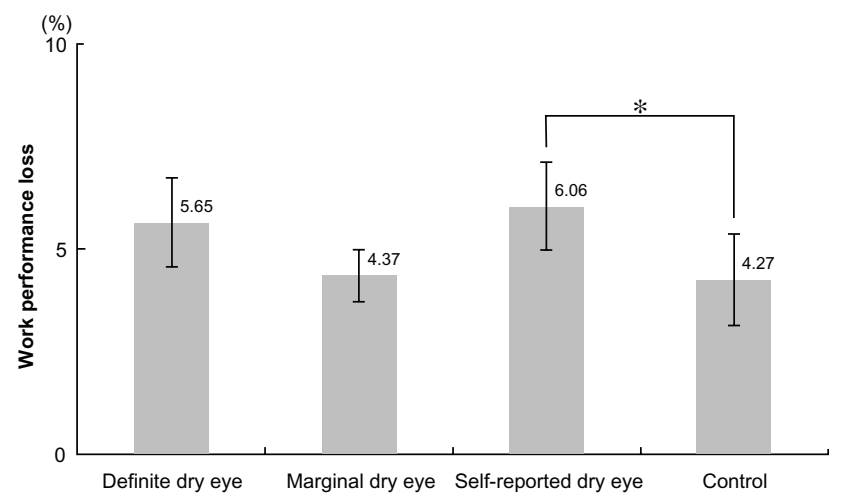

Figure I Work performance loss in each group.

Notes: The degree of work performance loss was $5.65 \%$ in the definite dry eye group, $4.37 \%$ in the marginal dry eye group, $6.06 \%$ in the self-reported dry eye group, and $4.27 \%$ in the control group. Productivity in the self-reported dry eye group was significantly lower than that in the control group. ${ }^{*} P<0.05$ (Dunnett's test). Error bar shows $95 \%$ confidence interval.

Moreover, although participants in both the definite dry eye group and the marginal dry eye group were diagnosed as having dry eye, they are different in terms of the presence of subjective symptoms. Therefore, we assumed that the difference in work performance loss between the definite dry eye group and the marginal dry eye group was due to impaired work performance because symptoms of dry eye were not controlled. Multiplying this difference (1.28\%) by the average annual wage resulted in USD 741. Given that it has been reported that the annual cost for treatment of dry eye
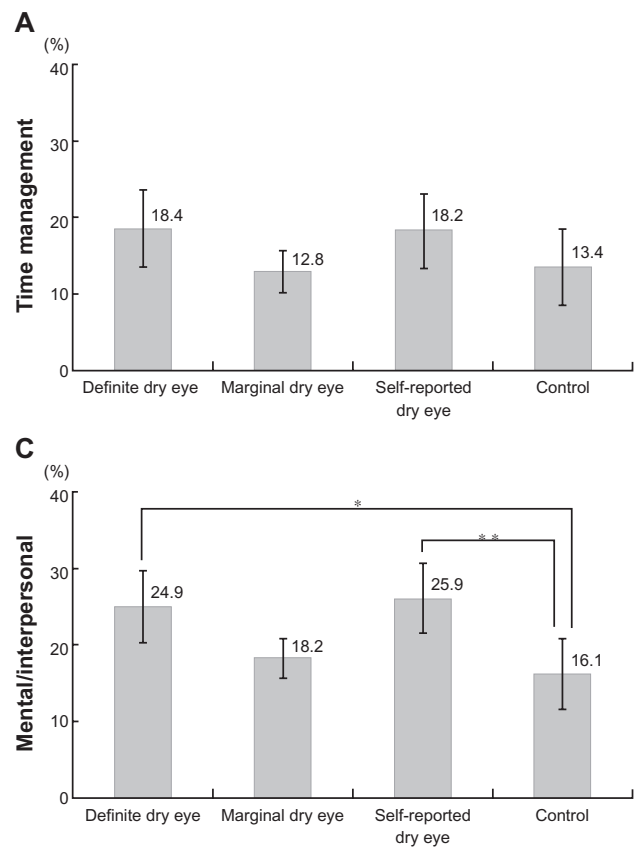

in Japan is USD $651,{ }^{19}$ it is shown that benefits of providing treatment for dry eye equal or outweigh the cost incurred by productivity loss.

\section{Discussion}

Available objective survey instruments to evaluate presenteeism include the WLQ, Stanford Presenteeism Scale, and Work Productivity and Activity Impairment Questionnaire, for each of which reliability and validity have been established. WLQ is a measurement tool developed by Lerner et al (Tufts Medical Center, Boston, MA) to assess work productivity loss associated with health problems, and has been used for chronic conditions, such as depression, osteoarthritis, back pain, migraine, and epilepsy. ${ }^{14-16}$

In the present study, we evaluated the impact of dry eye on presenteeism among office workers using WLQ-J. The degree of work performance loss by WLQ-J was $5.65 \%$ in the definite dry eye group and $6.06 \%$ in the self-reported dry eye group, showing higher scores compared with the control group, and there was a significant difference between the self-reported dry eye group and the control group. This revealed that subjective symptoms of dry eye led to an impairment in work performance among office workers. On the other hand, the degree of work performance loss in the marginal dry eye group was $4.37 \%$, which was comparable with that in the control group, suggesting that presenteeism

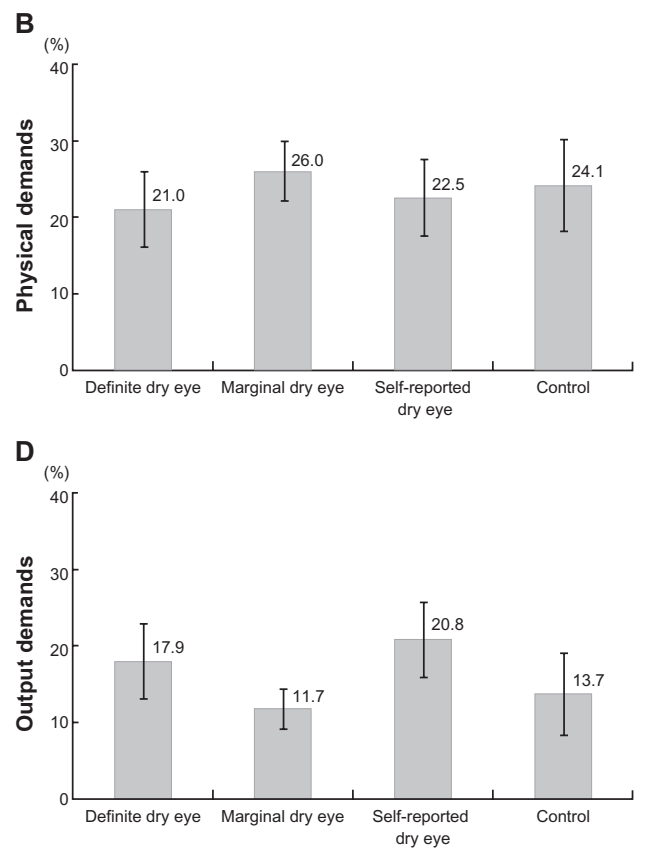

Figure 2 Work productivity loss by subscale scores for four aspects of work limitations, ie, time management (A), physical demands (B), mental/interpersonal (C), and output demands (D).

Notes: The mental/interpersonal score was significantly lower in the definite dry eye group $(P<0.05)$ and the self-reported dry eye group $(P<0.0 \mathrm{I})$. $* P<0.05$; $* * P<0.0 \mathrm{I}$ (one-way analysis of variance; Tukey or Games-Howell test was used for multiple comparisons). Error bar shows $95 \%$ confidence interval. Participants who had missing values ( 1 I in group I; 15 in group 2; four in group 3; II in group 4) were excluded from the analysis. 
may not be affected when subjective symptoms of dry eye have lessened due to treatment, even when the diagnosis has been established.

When work productivity loss was converted into an amount of money, the annual difference between the definite dry eye group and the control group was USD 799. This indicates that dry eye poses a significant disease burden on individual office workers. Moreover, the amount of money for work productivity loss caused by uncontrolled subjective symptoms as calculated by the difference between the definite dry eye group and the marginal dry eye group was USD 741 annually, which is equivalent to the annual medical treatment cost for treating dry eye in Japan of USD 651. This suggests that providing treatment to control symptoms of dry eye can be justified from the viewpoint of medical costs in addition to other benefits, such as enhancing daily function and improving quality of life.

According to longitudinal data from the labor force survey (February, 2011) issued by the Statistics Bureau, the Ministry of Internal Affairs and Communications, and a statistics training institution, the number of employees in Japan is estimated to be $62,940,000 .{ }^{20}$ Of these, $87.5 \%$ are office workers who use personal computers. ${ }^{21}$ Therefore, it is estimated that there are 55,070,000 office workers in Japan who use personal computers at work. Since the incidence of dry eye among visual display terminal workers in Japan has been reported to be between $23 \%{ }^{8}$ and $32.3 \%,{ }^{9}$ office workers with dry eye are estimated to number between $12,666,100$ and $17,790,000$. When the cost of work performance loss associated with uncontrolled dry eye is assumed to be USD 741 based on the difference between the definite dry eye group and the marginal dry eye group in the present study, the annual loss of productivity associated with dry eye in Japan is estimated to be USD 9386-15,386 million $(12,666,100-20,770,000 \times$ USD 741/year).

One of the clinical issues associated with dry eye is that many of the patients have not received medical management. ${ }^{1,2,22}$ In the present study, the degree of work performance loss was most prominent in the self-reported dry eye group, which included those who had symptoms of dry eye but who had not been diagnosed. This indicates that the consultation rate for patients with dry eye is generally low. Given that it is estimated that there are 12,666,100-20,770,000 office workers with dry eye in Japan, the economic benefits produced by medical treatment and subsequent increased productivity will reach USD 939-1539 million if $10 \%$ of the office workers with dry eye received medical treatment, and USD 4694-7693 million when 50\% of them received medical treatment (Figure 3).

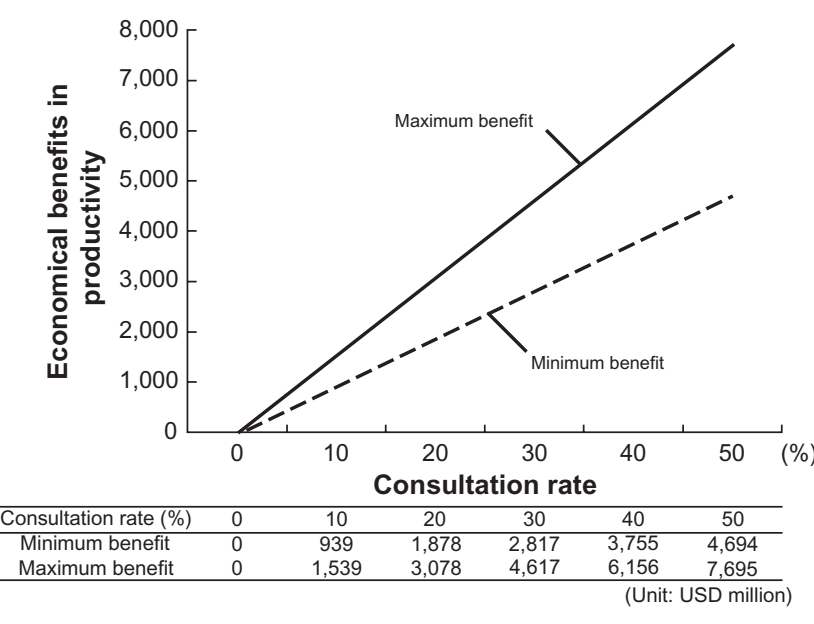

Figure 3 Economic benefits in productivity from providing treatment for dry eye. Note: When $50 \%$ of office workers with dry eye received treatment, the economic benefits were estimated to be USD 4694-7693 million.

The present study shows that office workers with symptoms of dry eye had work performance loss, which can lead to a substantial loss in work productivity. It was also shown that the medical cost for the treatment of dry eye outweighs the loss of productivity, producing economic benefits. Thus, it is very important to provide treatment for dry eye, because it not only improves quality of life for individual office workers, but also contributes to vitalization of the entire industry. In view of this, it may be important to enhance awareness of dry eye among office workers by educational activities.

\section{Disclosure}

The research was supported by a grant from Santen Pharmaceutical Company, Osaka, Japan. This study was presented in part at the 36th Japanese Cornea Conference, February 23-25, 2012, held in Tokyo, Japan.

\section{References}

1. Pflugfelder SC. Prevalence, burden, and pharmacoeconomics of dry eye disease. Am J Manag Care. 2008;14(Suppl 3):S102-S106.

2. Yamada M. Dry eye syndrome: concept, pathogenesis, and therapeutic modalities based on the new definition. J Jpn Ophthalmol Soc. 2009; 113(4):541-552. Japanese.

3. Schiffman RM, Christianson MD, Jacobsen G, Hirsch JD, Reis BL. Reliability and validity of the Ocular Surface Disease Index. Arch Ophthalmol. 2000;118(5):615-621.

4. Mertzanis P, Abetz L, Rajagopalan K, et al. The relative burden of dry eye in patients' lives: comparisons to a US normative sample. Invest Ophthalmol Vis Sci. 2005;46(1):46-50.

5. Mizuno Y, Yamada M, Miyake Y; Dry Eye Survey Group of the National Hospital Organization of Japan. Association between clinical diagnostic tests and health-related quality of life surveys in patients with dry eye syndrome. Jpn J Ophthalmol. 2010;54(4):259-265.

6. Miljanovic B, Dana R, Sullivan DA, Schaumberg DA. Impact of dry eye syndrome on vision-related quality of life. Am J Ophthalmol. 2007; 143(3):409-415. 
7. Schiffman RM, Walt JG, Jacobsen G, Doyle JJ, Lebovics G, Sumner W. Utility assessment among patients with dry eye disease. Ophthalmology. 2003;110(7):1412-1419.

8. Hikichi T, Yoshida A, Fukui Y, et al. Prevalence of dry eye in Japanese eye centers. Graefes Arch Clin Exp Ophthalmol. 1995;233(9): 555-558.

9. Uchino M, Schaumberg DA, Dogru M, et al. Prevalence of dry eye disease among Japanese visual display terminal users. Ophthalmology. 2008;115(11):1982-1988.

10. Rosenfield M. Computer vision syndrome: a review of ocular causes and potential treatments. Ophthalmic Physiol Opt. 2011;31(5):502-515.

11. Auren U. How to build presenteeism. Petroleum Refiner. 1955;34: 348-359.

12. Reddy P, Grad O, Rajagopalan K. The economic burden of dry eye: a conceptual framework and preliminary assessment. Cornea. 2004; 23(8):751-761.

13. Patel VD, Watanabe JH, Strauss JA, Dubey AT. Work productivity loss in patients with dry eye disease: an online survey. Curr Med Res Opin. 2011;27(5):1041-1048.

14. Lerner DJ, Amick B III; Glaxo Wellcome. Work Limitations Questionnaire. Boston MA: The Health Institute, Tufts-New England Medical Center; 1998.
15. Lerner DJ, Amick BC III, Rogers WH, Malspeis S, Bungay K, Cynn D. The Work Limitations Questionnaire. Med Care. 2001;39(1):72-85.

16. Lerner DJ, Reed JI, Massarotti E, Wester LM, Burke TA. The Work Limitations Questionnaire's validity and reliability among patients with osteoarthritis. J Clin Epidemiol. 2002;55(2):197-208.

17. Toda I, Fujishima H, Tsubota K. Ocular fatigue is the major symptom of dry eye. Acta Ophthalmol. 1993;71(3):347-352.

18. Japanese Ministry of Health, Labour and Welfare. [Basic Survey on Wage Structure 2010]. Available from: http://www.e-stat.go.jp/SG1/ estat/NewList.do?tid=000001011429. Accessed March 26, 2012. Japanese.

19. Mizuno Y, Yamada M, Shigeyasu C. Annual direct cost of dry eye in Japan. Clin Ophthalmol. 2012;6:755-760.

20. The Statistics Bureau. the Ministry of Internal Affairs and Communications. Long-term longitudinal data of the labor force survey. Tokyo, Japan. Feb, 2011. Japanese.

21. Japanese Ministry of Health, Labour and Welfare. Outline of the result of actual condition survey concerning technical innovation and labor. Tokyo, Japan. 2008. Japanese.

22. Shimmura S, Shimazaki J, Tsubota K. Results of a population-based questionnaire on the symptoms and lifestyles associated with dry eye. Cornea. 1999;18(4):408-411.
ClinicoEconomics and Outcomes Research

\section{Publish your work in this journal}

ClinicoEconomics \& Outcomes Research is an international, peerreviewed open-access journal focusing on Health Technology Assessment, Pharmacoeconomics and Outcomes Research in the areas of diagnosis, medical devices, and clinical, surgical and pharmacological intervention. The economic impact of health policy and health systems

\section{Dovepress}

organization also constitute important areas of coverage. The manuscript management system is completely online and includes a very quick and fair peer-review system, which is all easy to use. Visit http://www.dovepress.com/testimonials.php to read real quotes from published authors. 